\title{
Das Paragangliom - seltene Ursache einer mediastinalen Raumforderung
}

\author{
Paraganglioma - A Rare Cause of Mediastinal Lesions
}

Autoren

Institute
H. Knoop ${ }^{1}$, U. Knoop ${ }^{1}$, C. M. Heyer ${ }^{2}$, J. Reichert ${ }^{3}$, A. Tannapfel ${ }^{4}$, J. W. Walther ${ }^{1}$, G. Schultze-Werninghaus ${ }^{1 *}$

Die Institutsangaben sind am Ende des Beitrags gelistet. eingereicht 13.8 .2010

akzeptiert nach Revision 23. 8.2010

\section{Bibliografie}

DOI http://dx.doi.org/

10.1055/s-0030-1255763

Online-Publikation: 5. 10. 2010

Pneumologie 2010; 64:

752-754 ๔ Georg Thieme

Verlag KG Stuttgart · New York ISSN 0934-8387

\section{Korrespondenzadresse}

\section{Dr. Heiko Knoop}

Medizinische Klinik III -

Pneumologie, Allergologie,

Schlaf- und Beatmungsmedizin

Berufsgenossenschaftliches

Universitätsklinikum

Bergmannsheil GmbH

Klinikum der Ruhr-Universität

Bochum

Bürkle-de-la-Camp-Platz 1

44789 Bochum

Heiko.Knoop@

ruhr-uni-bochum.de

\section{Zusammenfassung \\ $\nabla$}

Eine 73-jährige Patientin wurde zur Abklärung einer unklaren mediastinalen Raumforderung in unserer Klinik vorgestellt. Wir führten endosonografisch eine Feinnadelpunktion durch. In der Zusammenschau aus Klinik, bildgebender Befunde und Zytologie entstand der hochgradige Verdacht auf ein Paragangliom. Diese Diagnose konnte letztlich nach kompletter chirurgischer Resektion histologisch bestätigt werden. Dieser Fallbericht über ein mediastinales Paragangliom gibt eine kurze Übersicht über das diagnostische Vorgehen und die therapeutischen Möglichkeiten dieser seltenen Erkrankung.

Eine 73-jährige Patientin wurde uns von ihrem Hausarzt zur Abklärung eines mediastinalen Tumors zugewiesen. Der Tumor war im Rahmen einer ambulant durchgeführten Computertomografie (CT) des Thorax aufgefallen. Diese wurde zur Abklärung einer Erhöhung der Blutsenkungsgeschwindigkeit (BSG) durchgeführt. BSG und Blutbild wurden bei der Patientin aufgrund einer durch gastroösophagealen Reflux bedingten Anämie mitZ.n. Fundoplicatio sowie eines Zustandes nach totaler Uterusextirpation und beidseitiger Adnexektomie 1992 aufgrund eines Androblastoms (Sertoli-Leydig-Zelltumor) regelmäßig kontrolliert. An sonstigen wesentlichen Vorerkrankungen bestand ein metabolisches Syndrom (arterielle Hypertonie, Hyperlipoproteinämie, Diabetes mellitus Typ 2, Adipositas mit einem BMI von $36,4 \mathrm{~kg} / \mathrm{m}^{2}$ ) und obstruktives Schlafapnoe-/-hypopnoesyndrom. Ein kausaler Zusammenhang zwischen erhöhter BSG und mediastinaler Raumforderung ist im Nachhinein auszuschließen: Die BSG normalisierte sich spontan und ließ sich am ehesten über einen entzündlichen Prozess im Bereich der Zähne erklären. Die Patientin äußerte bei ihrer Vorstellung in unserem Hause Wohlbefinden, insbesondere keine B-Symptomatik.

\section{Abstract \\ $\nabla$}

A 73-year-old female patient was admitted to our clinic for further clarification of a suspicious mediastinal lesion. Endosonographically, we performed a fine-needle aspiration biopsy. Clinical and radiological findings as well as cytology hardened the suspicion of a paraganglioma as being the cause of the mediastinal lesion. Finally, this diagnosis was confirmed histologically after complete surgical resection of the lesion. This case report on a mediastinal paraganglioma includes a concise summary of diagnostic procedures and therapeutic options for this rare disease.

In der CT des Thorax zeigte sich ein $45 \times 35 \mathrm{~mm}$ messender mediastinaler Tumor ( $\bullet$ Abb. 1).

Das Lungenparenchym war, abgesehen von einem unspezifischen subpleuralen Knötchen im apikalen Unterlappensegment rechts, unauffällig. Die Patientin wurde zur flexiblen Fiberbronchoskopie und Endosonografie stationär aufgenommen. Bronchoskopisch ergaben sich keine Tumorzeichen. In der transösophagealen Endosonografie (EUS, endoskopischer Ultraschall) zeigte sich eine echoreiche Raumforderung im aortopulmonalen Fenster $(\bullet$ Abb. 2).

Die Feinnadelpunktionszytologie zeigte Zellen eines neuroendokrinen Tumors, eine weitere zytologische Zuordnung gelang nicht. Ein Zusammenhang mit dem Sertoli-Leydig-Zelltumor ließ sich immunhistochemisch nicht belegen. In $\mathrm{Zu}-$ sammenschau aus Klinik, laborchemischen Befunden - insbesondere unauffälligen Lungen-assoziierten Tumormarkern und Beta2-Mikroglobulin -, Zytologie und Bildgebung wurde der hochgradige Verdacht auf ein Paragangliom geäußert. Zum Ausschluss weiterer Raumforderungen wur-

\footnotetext{
* Die Autoren danken der Praxis „Radiologie-EnnepeRuhr“ für die freundliche Überlassung des CT-Bildes.
} 


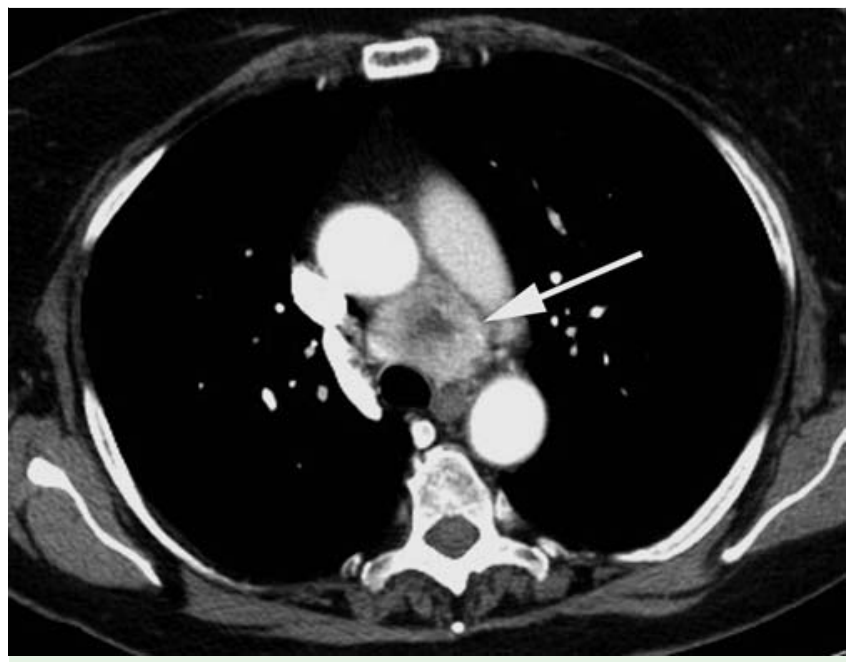

Abb. 1 In der kontrastverstärkten Thorax-CT demarkiert sich eine im zentralen Mediastinum dorsal der Aorta descendens gelegene, solide Raumforderung (Pfeil) mit einer Größe von $45 \times 35$ mm. Der Tumor zeigt ein deutliches, peripher betontes Enhancement als Ausdruck der kräftigen Durchblutung.

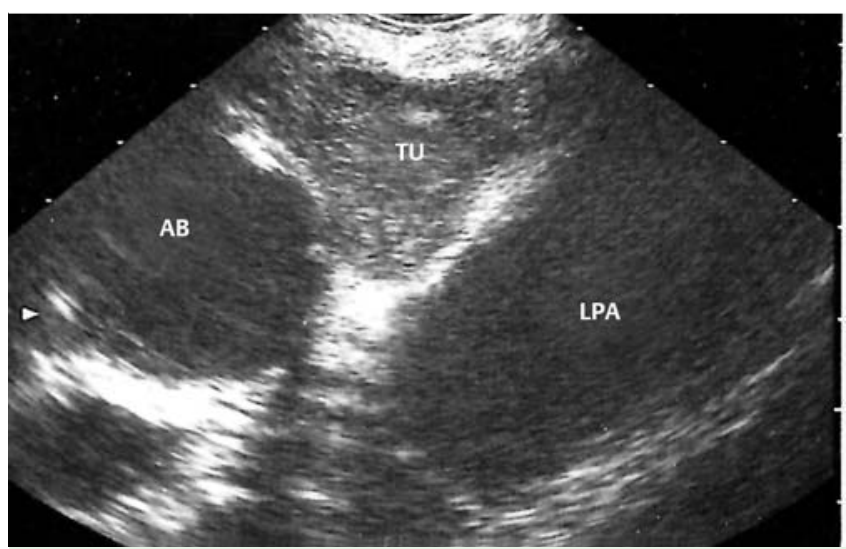

Abb. 2 Im EUS zeigt sich der Tumor (TU) im aortopulmonalen Fenster $(\mathrm{AB}=$ Aortenbogen, $\mathrm{LPA}=$ linke Pulmonalarterie $)$.

de eine Octreotid-Szintigrafie angeschlossen. Diese zeigte, abgesehen von der Nuklidmehrbelegung im Bereich der mediastinalen Raumforderung, keinen pathologischen Befund ( $\bullet$ Abb. 3).

Die Patientin wurde daher zeitnah zur Tumorexstirpation in die Klinik für Herz-Thoraxchirurgie aufgenommen.

Intraoperativ zeigte sich ein abgekapselter Tumor, der derbe mit dem umliegenden Gewebe verwachsen und extrem vulnerabel war. Nach makroskopischen Aspekten konnte der Tumor in toto entfernt werden. Die histologische Aufarbeitung bestätigte ein sympathisches Paragangliom ( $\bullet$ Abb. 4) mit typischer immunhistochemischer Analyse (S-100 positiv) ( $\bullet$ Abb. 5).

Bei fehlenden Infiltrationszeichen in Blut- oder Lymphgefäßen und nicht vorhandenen Nekrosen oder atypischen Mitosen gehen wir von einem gutartigen Verlauf aus. Ebenfalls entfernte mediastinale Lymphknoten waren tumorfrei. Postoperativ war die Patientin unauffällig. Eine weitere Therapie musste aufgrund der R0-Situation nicht angeschlossen werden. Klare Empfehlungen zum Follow-up lassen sich in der Literatur wegen der Seltenheit des Tumors nicht finden: Wir haben nach einem sechsmonatigen Zeitintervall eine computertomografische Verlaufskontrolle empfohlen.

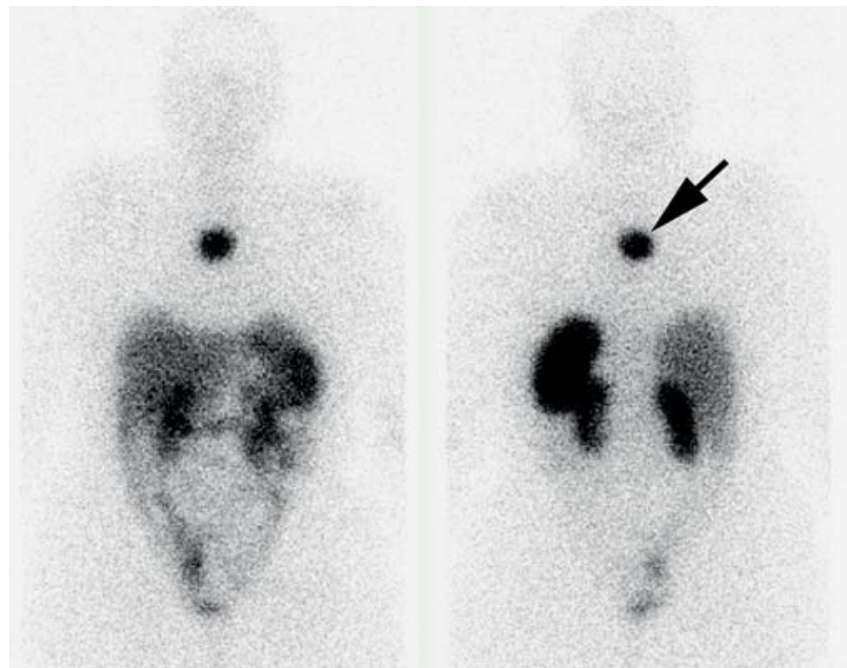

Abb. 3 Nach Gabe von $145 \mathrm{MBq}$ Indium 111 Somatostatin (an ${ }^{111}$ Indium gekoppeltes Octreotid) zeigt sich in der Ganzkörperszintigrafie eine kräftige Mehrbelegung (Pfeil) im zentralen Mediastinum bei ansonsten physiologischer Mehrbelegung in Leber, Milz, Nieren und Darm, hinweisend auf einen Somatostatin-Rezeptor-positiven Tumor.

Paragangliome sind seltene Tumoren, die aus Paraganglien hervorgehen. Paraganglien bestehen aus epithelähnlichen Parenchymzellen, einem gefäßreichen Interstitium und sind von einer Bindegewebskapsel umgeben. Sie entstehen im Rahmen der fetalen Entwicklung aus Zellen, die aus der Neuralleiste vor allem nach paraaortal migrieren [1]. Nach der Anfärbbarkeit unterscheidet man chromaffine und nicht chromaffine Paraganglien, nach der Herkunft sympathische und parasympathische. Beim Fetus liegt das meiste chromaffine Gewebe in Paraganglien, die physiologischerweise nach der Geburt innerhalb von zwei bis drei Jahren vollständig atrophieren. Paragangliome können theoretisch an jeder Stelle des Migrationsweges der spezialisierten Neuralleistenzellen entstehen und Neuropeptide und Katecholamine sezernieren. Sie werden nach ihrer anatomischen Lokalisation und Innervation als brachiomer, intravagal, aortikosympathisch und viszeroautonom bezeichnet. $\mathrm{Zu}$ den brachiomeren Paragangliomen, die eine Beziehung zu den Hirnnerven haben, zählen unter anderem auch die aortopulmonalen.

Paragangliome können familiär gehäuft vorkommen [2]. Der Erbgang ist autosomal-dominant. Die häufigsten Mutationen scheinen die Untereinheit D des Succinat-Dehydrogenase-Komplexes - einem Bestandteil der mitochondrialen Atmungskette zu betreffen [3].

Die Freisetzung von vasoaktiven Katecholaminen durch den Tumor kann klinisch zu arterieller Hypertonie, Palpitationen, Kopfschmerzen, Gewichtsverlust und profusem Schwitzen führen. Als bildgebendes Verfahren ist die Computertomografie das Verfahren der Wahl zur Bestimmung der Ausdehnung der Tumormasse und Angrenzung an umliegende anatomische Strukturen. Bei Verdacht auf ein Paragangliom im Bereich der Karotiden sollte die Diagnostik ferner um eine Dopplerultraschalluntersuchung dieser Region ergänzt werden [4]. Insbesondere im Kopf-HalsBereich ist die Magnetresonanztomografie mit Kontrastmittelgabe dem CT hinsichtlich der Abgrenzbarkeit gegenüber umliegenden Gewebs- und Gefäßstrukturen überlegen. Szintigrafien mit dem Somatostatin-Analogon Octreotid dienen dazu, Tumor- 


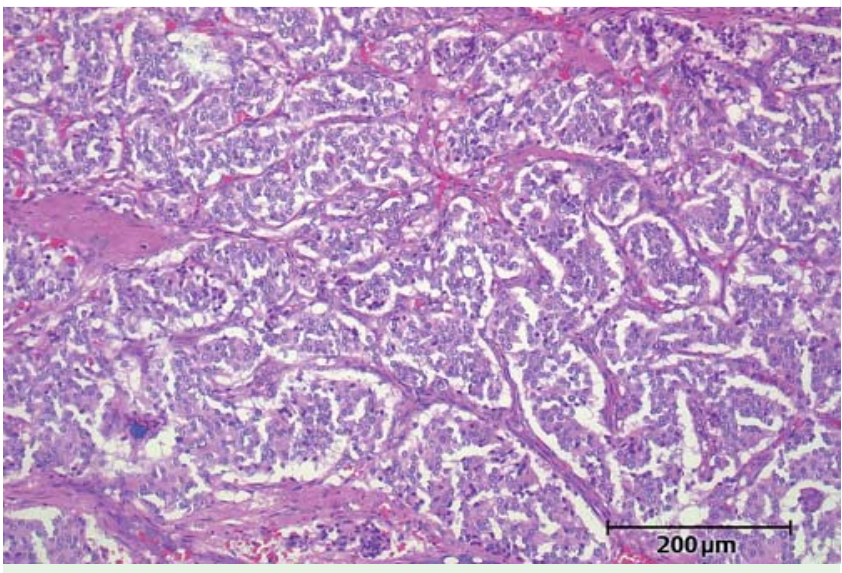

Abb. 4 Hämatoxylin-Eosin(HE)-Bild (Originalvergrößerung siehe Maßstab): typisches histologisches Bild von monomorphen, netzartig und ballenförmig angeordneten neuroendokrinen Zellen. Die Zellen mit hellem Zytoplasma, die Zellkerne ohne relevante Atypien und zellzentraler Anordnung. Die ballenförmig angeordneten Zellen werden von fibrovaskulären Septen umgeben.

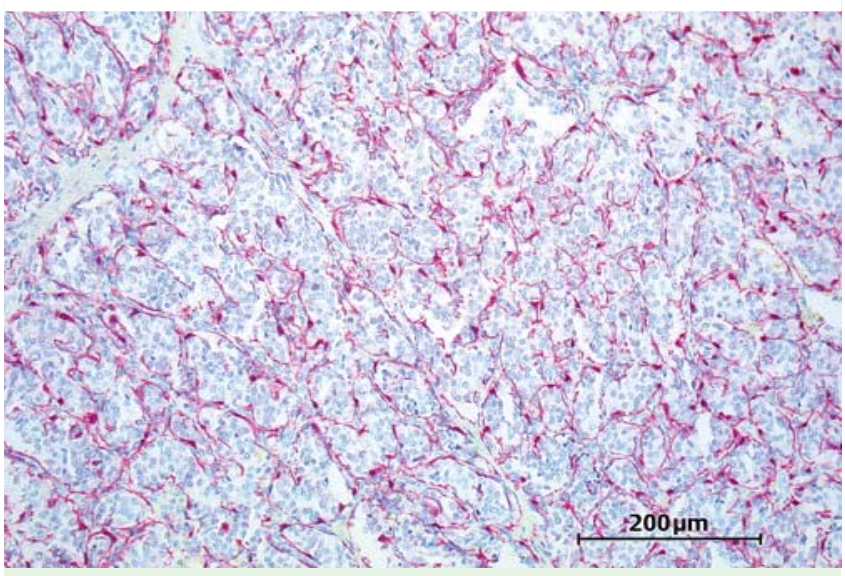

Abb. 5 In der S-100-Markierung zeigen sich die typischen positiv markierten Sustentikularzellen. Die Sustentikularzellen sind Stützzellen, die mittels ihrer Zellfortsätze ein Netzwerk mit stacheldrahtähnlicher Morphologie bilden. Die Prävalenz dieser Zellen korreliert in adrenalen und extraadrenalen Paragangliomen invers mit der Prognose der Patienten.

manifestationen des Paraganglioms in weiteren Organsystemen sichtbar zu machen [5]. Hierbei macht man sich die hohe Dichte von Somatostatin-2-Rezeptoren an der Oberfläche des Paraganglioms zu Nutze. In der Regel gelingt die Diagnosestellung über die bildgebenden Befunde und klinischen Angaben. Eine Biopsie ist nicht zwingend erforderlich und birgt aufgrund der hohen Vaskularisierung des Tumors ein gewisses Blutungsrisiko.

In der immunhistochemischen Gewebsanalyse werden unter anderem Antikörper gegen S-100 eingesetzt: S-100 bezeichnet eine Multigenfamilie, die für Calcium-bindende Proteine mit geringer Molekülmasse und vielfältigen Zellfunktionen kodiert. Die Anfärbbarkeit gegen S-100 ist ein Unterscheidungsmerkmal zur Charakterisierung von Tumorzellen. Darüber hinaus werden Parameter wie S-100-Negativität, Nekrosen, Mitosen und Zellpolymorphismen als Malignitätskriterien zu Rate gezogen. Sie sind allerdings unzuverlässig.

Weniger als 10\% der Paragangliome sind maligne [6]. Malignität ist abhängig von der Lokalisation [6]. Risikofaktor für Malignität ist eine familiäre Disposition (SDH B-Mutation) [3]. Ein systematisches genetisches Screening auf SDH B-Mutationen bei Patienten mit Paragangliom wird dennoch nicht empfohlen. Neben einem malignen Verlauf und dem damit verbundenen möglichen Auftreten von Metastasen ist die lokale Kompression durch das Tumorwachstum eine weitere Hauptkomplikation des Paraganglioms insbesondere auch beim mediastinalen Paragangliom [6]. Therapie der Wahl ist dementsprechend die vollständige chirurgische Resektion. Vorherige Arteriografie und Embolisation kann zu einer Verminderung der Blutungskomplikation beitragen. Intraoperativ sollten ipsilaterale Lymphknoten wegen des potenziellen Malignitätsrisikos mit entfernt werden. Die Radiatio kommt insbesondere bei Inoperabilität zum Einsatz. Die Bedeutung einer adjuvanten Bestrahlung ist nicht hinreichend belegt [7]. Bei fortgeschritten metastasierten Paragangliomen wird eine Chemotherapie empfohlen, ohne dass sich ein bestimmtes Protokoll als überlegen herausgestellt hätte. Üblicherweise kommen Cyclophosphamid, Dacarbazin, Vincristin mit oder ohne Kombination mit Doxorubicin zum Einsatz [8].

Metastasen können noch bis zu 20 Jahre nach der operativen Sanierung auftreten, sodass konsequente Nachuntersuchungen obligat sind [9]. Prinzipiell ist die Prognose nach kompletter Resektion aber günstig [10].

\section{Interessenkonflikt}

Die Autoren geben an, dass kein Interessenkonflikt besteht.

\section{Institute}

Medizinische Klinik III - Pneumologie, Allergologie, Schlaf- und Beatmungsmedizin, Berufsgenossenschaftliches Universitätsklinikum Bergmannsheil GmbH (Direktor: Prof. Dr. G. Schultze-Werninghaus)

2 Institut für Diagnostische und Interventionelle Radiologie und Nuklearmedizin, Berufsgenossenschaftliches Universitätsklinikum Bergmannshei GmbH (Direktor: Prof. Dr. V. Nicolas)

Klinik für Herz- und Thoraxchirurgie, Berufsgenossenschaftliches Universitätsklinikum Bergmannsheil GmbH (Direktor: Prof. Dr. A. Laczkovics)

4 Institut für Pathologie der Ruhr-Universität Bochum an der Berufsgenossenschaftlichen Universitätsklinik Bergmannsheil (Direktorin: Prof. Dr. A. Tannapfel)

\section{Literatur}

1 Rohen JW, Lütjen-Drecoll E. Funktionelle Embryologie. Stuttgart: Schattauer, 2006: 56-59

2 Drovdlic CM, Myers EN, Peters JA et al. Proportion of heritable paraganglioma cases and associated clinical characteristics. Laryngoscope 2001; 111: $1822-1827$

3 Boedeker CC, Neumann HP, Maier Wet al. Malignant head and neck paragangliomas in SDHB mutation carriers. Otolaryngol Head Neck Surg 2007; 137: $126-129$

4 Stoeckli SJ, Schuknecht B, Alkadhi $H$ et al. Evaluation of paragangliomas presenting a cervical mass on color-coded Doppler sonography. Laryngoscope 2002; 112: $143-146$

5 Telischi FF, Bustillo A, Whiteman ML et al. Octreotide scintigraphy for the detection of paragangliomas. Otolaryngol Head Neck Surg 2000; 122: $358-362$

6 Brown ML, Zayas GE, Abel MD et al. Mediastinal paragangliomas: The Mayo Clinic Experience. Ann Thorac Surg 2008; 86: 946 - 951

7 Lee JH, Barich F, Karnell LH et al. National Cancer Data Base report on malignant paragangliomas of the head and neck. Cancer 2002; 94: $730-737$

8 Patel SR, Winchester DJ, Benjamin RS. A 15-year experience with chemotherapy of patients with paraganglioma. Cancer 1995; 76: 1476-1480

9 Hinerman RW, Amdur RJ, Morris CG et al. Definitive radiotherapy in the management of paragangliomas arising in the head and neck: a 35year experience. Head Neck 2008; 30: 1431-1438

10 Erickson D, Kudva YC, Ebersold MJ et al. Benign paragangliomas: clinical presentation and treatment outcomes in 236 patients. J Clin Endocrinol Metab 2001; 86: 5210-5216 